\title{
Stratégies d'occupation de l'espace par les petits ligneux après débroussaillement en région méditerranéenne française. Exemple d'un réseau de pare-feu dans l'Esterel
}

\author{
M Étienne, C Legrand, D Armand
}

\author{
INRA, BP 91, Unité d'écodéveloppement, 84143 Montfavet, France
}

(Reçu le 21 mars 1991; accepté le 21 juin 1991)

\begin{abstract}
Résumé - La repousse de la strate arbustive de 6 fruticées typiques de peuplements forestiers du massif de l'Esterel a été suivie pendant 6 ans sur des pare-feu créés il y a plus de 20 ans, et régulièrement entretenus depuis. II s'agit de faciès à cistes (Cistus salviaefolius, $C$ monspeliensis et $C$ albidus), à bruyères (Erica arborea et $E$ scoparia), à myrte (Myrtus communis), à callune (Calluna vulgaris), à calycotome (Calycotome spinosa) et à cytise triflore (Cytisus triflorus). L'analyse de l'accroissement en volume et en poids montre des stratégies d'occupation de l'espace contrastées selon les types de peuplement, avec des faciès à fort encombrement, faible phytomasse et colonisation rapide (cytise, calycotome); des faciès à faible encombrement, forte phytomasse et colonisation rapide (callune); et des faciès à faible encombrement, faible phytomasse et colonisation lente (cistes).
\end{abstract}

embroussaillement / pare-feu / phytomasse / phytovolume / vitesse / région méditerranéenne

Summary - Spatial occupation strategy of shrublands after cutting in the French Mediterranean region. Growth rate of shrubs after cutting is the determining factor when programming scrub clearing schedules in fuel-break networks, especially when their maintenance is partly carried out by livestock grazing. As vegetation grows quickly on acid soils, 6 types of shrublands were studied for 6 years in the Esterel hills (southeastern France). These plant communities were growing in 20-yearold fuel-breaks (cut regularly) and were dominated by Calluna vulgaris in the first Calycotome spinosa in the second, Cytisus triflorus in the third, Erica arborea and E scoparia in the fourth, Myrtus communis and Pistacia lentiscus in the fifth and Cistus salviaefolius, $\mathrm{C}$ monspeliensis and $\mathrm{C}$ albidus in the last. A permanent belt transect $20 \mathrm{~m}$ long and $50 \mathrm{~cm}$ wide was measured every year in June, just after the spring growth. This method gave brushwood amount, fuel stock and browse biomass. $A$ volumetric space factor was used to estimate brushwood amount and aerial biomass was calculated to evaluate fuel stock. Multiple regression models (table I) classified the shrublands into 3 groups according to biomass or volume growth. An index based on biomass corrected by a coefficient inversely proportional to the initial shrubland cover classified shrublands in relation to their fuel production potential (table II). Volume data are compared with the current volume in the controls (table III) and with the regression models of the dominant species (table V). Changes in the dominant species ratio are also commented on (table IV). The analysis of biomass and volume growth rates gave 2 oppo- 
sing strategies: Calluna and Cytisus stands sprouted vigorously and then their growth rate decreased steadily, while the other stands, after a slow start increased their growth rate constantly until the fourth year (fig 1). A preliminary growth rate model grouping all the stands with similar dominant species is proposed (fig 2). Altogether, each type of shrubland can be characterized by a particular strategy of spatial occupation: Cytisus or Calycotome stands developed large volume, light biomass and rapidly spreading shrublands; Calluna formed low volume, heavy biomass and rapidly spreading heathlands; while Cistus dominance generated low volume, light biomass and slowly spreading rangelands.

shrub encroachment / fire-break / blomass / bulk volume / growth rate / Mediterranean region

\section{INTRODUCTION}

Les premières actions de protection de la forêt méditerranéenne française contre les incendies, lancées dans les années 70 , se sont concrétisées par la création de grands périmètres de pare-feu, puis ont été suivis, au cours de ces dernières années, par la mise en place de PIDAF*. L'ensemble de ce réseau couvre de vastes surfaces qui sont périodiquement débroussaillées manuellement ou mécaniquement.

Dans la dynamique de nombreux groupements végétaux méditerranéens, les stades pionniers de recolonisation de l'espace par la pelouse sont souvent extrêmement éphémères car ils sont rapidement masqués par le développement des espèces arbustives. Ceci est particulièrement vrai sur sol pauvre ou quand l'ouverture du peuplement initial est due à un feu ou à un débroussaillement sans travail du sol.

L'effet de ces coupes répétées sur la dynamique des peuplements végétaux débroussaillés est fort peu connue alors que des informations sur les modalités et les vitesses de régénération des espèces ligneuses devraient permettre de mieux ajuster la périodicité des interventions ou même de choisir la technique de débrous- saillement la plus efficace vis-à-vis du milieu concerné.

Dans ce but, la dynamique de peuplements arbustifs variés a été suivie pendant 6 ans sur un réseau de pare-feu de l'Esterel, dans le Sud-Est de la France.

\section{MATÉRIEL ET MÉTHODE}

Il s'agit de forêts de pin pignon ou de chêne liège poussant sur des sols faiblement ondulés issus de grès rouges du Permien et sous un climat méditerranéen de type subhumide frais. Le sous-bois a été débroussaillé mécaniquement tous les 2-3 ans depuis une vingtaine d'années; le point de départ de nos mesures est donc un stade régressif de maquis ayant subi 4-5 coupes et présentant des recouvrements initiaux compris entre 30 et $50 \%$, sauf pour la formation à bruyères qui correspond à un pare-feu d'ouverture récente. L'ensemble du dispositif est pảturé par un troupeau de moutons avec une intensité de 300-400 journées-brebis/ha. Trois des faciès étudiés ont fait l'objet d'améliorations pastorales par fertilisation of sursemis depuis 3 ans, et sont soumis à une pression de pâturage plus élevée à partir de cette date (1 500$2000 \mathrm{jb}^{2} \mathrm{ha}^{-1}$ ).

L'étude a été effectuée pendant 5 ans au mois de juin sur 10 sites couvrant 5 faciès : une cistaie avec 2 stations sursemées et une non sursemée, une myrtaie et un faciès à cytise avec 2 répétitions, une ériçaie fertilisée, une cal-

* Plan intercommunal de débroussaillement et d'aménagement forestier. 
lunaie et un maquis à calycotome. Les espèces dominant ces faciès avant le dernier débroussaillement étaient :

- Cistus salviaefolius L, C monspeliensis L, C albidus $\mathrm{L}$ et Lavandula stoechas $\mathrm{L}$ pour les cistaies;

- Myrtus communis L, Phillyrea angustifolia L et Pistacia lentiscus L pour les myrtaies;

- Cytisus triflorus L'Héritier ou Calycotome spinosa (L) Link pour les maquis à cytise ou à calycotome;

- Erica arborea L, E scoparia L, Pistacia lentiscus $L$ et Phillyrea angustifolia $L$ pour l'ériçaie;

- Calluna vulgaris (L) Hull pour la lande à callune.

Le recouvrement et le phytovolume total et par espèce ont été mesurés par la méthode du transect en bande (Étienne et Legrand, 1991) sur 5 types de maquis. La méthode consiste à repérer la projection des différentes strates de végétation le long d'une bande de $20 \mathrm{~m}$ couvrant $10 \mathrm{~m}^{2}$, chaque touffe ainsi repérée étan renseignée par le nom et la hauteur des espèces correspondantes. Le volume d'encombrement a été calculé en multipliant la surface de la projection de l'arbuste par sa hauteur. II a ensuite été transformé en phytomasse à partir des données de densité moyenne établies par ailleurs au moyen d'un double échantillonnage (Baudin, 1985).

\section{RÉSULTATS}

\section{Volume et phytomasse par faciès}

Des modèles de croissance en volume et en phytomasse ont été recherchés pour chacun des faciès. Dans tous les cas, d'excellents résultats ont été obtenus, avec des régressions de type puissance (tableau 1).

\section{Phytovolume aérien global}

Nous pouvons distinguer plusieurs familles de courbe selon les modalités d'encombrement de l'espace des peuplements arbustifs (tableau I). La première regroupe les faciès à croissance lente, cistaies et callunaie, caractérisés par des exposants compris entre 1,7 et 1,9 et des phytovolumes

Tableau l. Équations de régression du phytovolume (m3/ha) et de la phytomasse (kgMS/ha) en fonction de l'âge (mois) pour 10 formations arbustives de l'Esterel.

\begin{tabular}{|c|c|c|c|c|}
\hline Formation & Volume $=f(a \hat{g} \theta)$ & $r$ & Poids $=f(\hat{a g} \theta)$ & $r$ \\
\hline Ciste clair ${ }^{* *}$ & $Y=0,78 X^{1,69}$ & 0,99 a & $Z=0,84 X^{1,83}$ & $0,99 \circ$ \\
\hline Ciste dense ** & $Y=1,04 X^{1,8}$ & 0,99 a & $Z=1,1 X^{1,93}$ & $0,99 a$ \\
\hline Callune ${ }^{* * *}$ & $Y=1,36 X^{1,82}$ & 0,99 a & $Z=1,4 \times 1,95$ & $0,98=$ \\
\hline Lavande ciste ${ }^{* *}$ & $Y=0,77 X^{1,88}$ & 0,98 a & $Z=0,79 \times 1,99$ & $0,98^{\circ}$ \\
\hline Myrte filaire ${ }^{* \star}$ & $Y=1,1 \times 1,98$ & 0,99 a & $Z=1,02 X^{2,12}$ & $0,99=$ \\
\hline Cytise ** & $Y=1,98 X^{2}$ & $0,96 \mathrm{~b}$ & $Z=1,72 X^{1,76}$ & $0,97 b$ \\
\hline Cytise fougère ** & $Y=1,79 X^{2,01}$ & $0,97^{b}$ & $Z=1,6 \times 1,6$ & 0,97 \\
\hline Bruyère myrte * & $Y=1,23 X^{2,03}$ & $0,99 \mathrm{c}$ & $Z=1,2 X \quad 2,15$ & 0,99 \\
\hline Calycotome ** & $Y=1,41 X^{2,09}$ & 0,99 a & $Z=1,2 \times 1,89$ & $0,99=$ \\
\hline Bruyère * & $Y=1,09 X^{2,78}$ & $0,99^{b}$ & $Z=1,13 \times 2,38$ & 0,99 \\
\hline
\end{tabular}

Régressions calculées sur ${ }^{*} 3,{ }^{* *} 5$ ou ***7 années; significatives au seuil de a: $P<0,001 ; \mathrm{b}: P<0,01 ; \mathrm{c}: P<0,1$. 
atteignant 700 à $1800 \mathrm{~m}^{3} / \mathrm{ha}$ au bout de 5 ans. La seconde, avec des exposants de 2 à 2,1 regroupe les faciès à myrte, cytise et calycotome, atteignant des volumes de 3300 à $4800 \mathrm{~m}^{3 / h a}$ à 5 ans. L'ériçaie se distingue avec un exposant de 2,8 et un phytovolume de $3000 \mathrm{~m}^{3} /$ ha dès 3 ans.

\section{Phytomasse aérienne globale}

De la même manière, il est possible d'associer à un faciès donné une loi de croissance en poids, caractérisée par l'exposant de la régression (tableau 1). Les faciès à cytise présentent les croissances les plus faibles, avec des exposants compris entre 1,6 et 1,8 du fait de la faible masse volumique de leur espèce dominante. Les cistaies constituent encore des peuplements peu productifs, comme la callunaie et le faciès à calycotome, en suivant des courbes dont les exposants restent inférieurs à 2. Les myrtaies et l'ériçaie forment un troisième groupe avec des exposants supérieurs à 2,1 qui démontrent une accumulation soutenue de phyto- masse au cours des 3 premières années de repousse.

\section{Phytomasse avant et après débroussaillement}

Les maquis étudiés ayant subi la même séquence de débroussaillements, il est intéressant de comparer la quantité de phytomasse produite au bout d'un temps donné dans les différents faciès avec la phytomasse existant juste avant la dernière coupe (tableau II). La périodicité de nettoyage des pare-feu dans les réseaux gérés par l'Office national des forêts étant de 2 à 3 ans, nous avons choisi un pas de temps de 3 ans pour effectuer ces comparaisons.

Si l'on interprète ces données en terme de récupération de la production primaire, on observe qu'au bout de 3 ans, les gros maquis à cytise, calycotome ou bruyère ont déjà dépassé leur phytomasse initiale. Leur vigueur ne semble donc pas du tout affectée par le débroussaillement, ce qui

Tableau II. Phytomasse à 3 ans (TMS/ha) après la dernière coupe (P3) en relation avec le recouvrement arbustif (Ri en \%) et la phytomasse (Pi) avant le dernier débroussaillement.

\begin{tabular}{llll} 
Faciès & $P i$ & $P 3$ & $P 3 / R i$ \\
\hline Ciste dense & $3,1^{*}$ & 1,1 & 2,2 \\
Ciste clair & $2^{*}$ & 0,7 & 2,3 \\
Cytise fougère & - & 0,5 & 2,5 \\
Cytise & $0,7^{*}$ & 0,8 & 2,7 \\
Calycotome & $0,6^{* *}$ & 0,8 & 2,7 \\
Lavande ciste & - & 1,7 & 4,3 \\
Myrte filaire & - & 2,4 & 4,8 \\
Callune & - & 2,7 & 5,4 \\
Myrte bruyère & - & 2 & 5,7 \\
Bruyère & $3,6^{* *}$ & 4,7 & 11,8 \\
& & & \\
\hline
\end{tabular}

Faciès âgé de ${ }^{*} 2$ ou **3 ans. 
est compréhensible pour la bruyère qui n'en est qu'à sa deuxième coupe, mais est assez surprenant pour les calycotomes et les cytises qui ont déjà été traités 5 fois. Cette puissance de rejet est probablement liée à la capacité qu'ont ces espèces de mettre en réserve de grandes quantités de carbohydrates dans leur racine pivotante ou dans leur lignotuber (Vinck, 1987; Mesleard, 1987).

Pour comparer les faciès entre eux, il est nécessaire de gommer l'effet lié aux différences de recouvrement entre stations. Nous avons pour cela appliqué aux données de phytomasse un facteur correcteur inversement proportionnel au recouvrement du peuplement arbustif avant coupe (tableau II). Si l'on ordonne ces phytomasses corrigées par ordre croissant, on voit apparaître 5 groupes de faciès.

Les cistaies sursemées présentent les phytomasses corrigées les plus faibles et n'ont toujours pas retrouvé leur phytomasse initiale au bout de 5 ans. Étant donné le faible effet du pâturage sur les espèces arbustives qui les composent (Combier, 1990), ce phénomène est probablement lié à la concurrence du tapis herbacé introduit. Ce résultat confirme les dynamiques observées en Corse sous l'effet du développement d'un tapis dense de trèfle souterrain spontané après fertilisation (Étienne, 1977a; Joffre et Casanova, 1983). Les vitesses de récupération des cistaies y sont du même ordre, avec une repousse arbustive de seulement 500 $\mathrm{kgMS} / \mathrm{ha}$ au bout de 2 ans, dans les parcelles fertilisées, contre $2 \mathrm{~T}$ dans celles simplement débroussaillées (Étienne, 1977b).

Les maquis à calycotome ou à cytise constituent également un groupe à faible indice de phytomasse. Ceci est dû à la très faible masse volumique sèche des espèces dominantes, leur valeur $(0,36$ et
$0,39 \mathrm{~kg} / \mathrm{m}^{3}$ respectivement) étant $5-6$ fois moindre que celle des autres espèces considérées (Baudin, 1985).

Viennent ensuite la cistaie non sursemée, la callunaie et les myrtaies qui, avec des indices allant de 4 à 6 , forment un ensemble de maquis vigoureux se régénérant aussi bien par graine que par rejet de souche.

Enfin, l'ériçaie se distingue nettement avec une production corrigée moyenne approchant $4 \mathrm{TMS} / \mathrm{ha} / \mathrm{an}$. Ce chiffre énorme s'explique d'une part, par la puissance de rejet des espèces qui constituent ce type de maquis et d'autre part, par le fait que ce faciès n'a subi que 2 débroussaillements, car il correspond à des pare-feu récemment créés sur des peuplements âgés d'environ 35 ans.

\section{Comparaison avec des maquis adultes}

Les phytovolumes atteints 5 ans après coupe peuvent être rapprochés de chiffres obtenus sur des parcelles voisines, n'ayant subi aucune perturbation depuis une trentaine d'années, et considérées comme "témoins". Les résultats obtenus sur des ériçaies et des cistaies montrent que plus de la moitié du volume d'encombrement du maquis initial est retrouvée dans ce délai (tableau III).

\section{Modification de la composition botanique}

Nous avons voulu vérifier de quelle façon l'effet de la coupe et/ou du pâturage pouvait modifier la composition des peuplements arbustifs étudiés. Le faciès bruyère illustre parfaitement l'effet combiné de ces 2 facteurs (tableau IV). On observe en effet que les cistes deviennent progressi- 
Tableau III. Phytovolume (V) et recouvrement (R) de maquis adultes, et part respective des espèces.

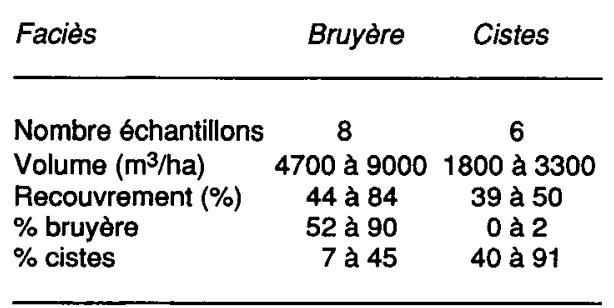

vement dominants et que le lentisque est la seule espèce se développant par rejet à se maintenir. Ceci est en opposition avec les données de la littérature où les espèces rejettant de souche sont considérées comme étant favorisées après perturbation (Keeley et Zedler, 1979; Johnson et al, 1986). En fait, l'impact du pâturage se superpose à celui de la coupe en affectant les espèces ligneuses proportionnellement à leur appétence; la filiaire est totalement consommée, et la bruyère arborescente est fortement touchée (Combier, 1990).

La stratégie de substitution ou d'occupation des espaces vides par les cistes se retrouve sur d'autres faciès, mais il faut si- gnaler que des phénomènes de concurrence se développent également entre espèces de ciste. Ainsi, dans la "cistaie claire", on constate une substitution progressive du ciste de Montpellier par le ciste à feuille de sauge. Ce phénomène est probablement lié à la capacité de ce dernier à se ramifier après une coupe, grâce à la présence de bourgeons dormants situés juste au-dessus du collet. Le développement du ciste blanc au détriment des cistes précédemment cités dans la cistaie dense est plus difficile à expliquer.

Signalons enfin que la régression des maquis s'accompagne toujours d'une progression des petits ligneux aromatiques comme Lavandula stoechas ou Thymus vulgaris, mais l'impact de cette colonisation sur le degré de salissement des parefeu est tout à fait négligeable.

\section{Seuil de débroussaillement}

Les praticiens (forestiers, professionnels de la DFCI), ont l'habitude d'estimer visuellement le seuil à partir duquel un pare-feu ne remplit plus sa fonction et doit être débroussaillé. Nous avons pu établir que ce

Tableau IV. Évolution de la part relative des espèces au volume total sur le transect bruyère.

\begin{tabular}{lrrrr}
\hline & 1986 & 1987 & 1988 & 1989 \\
\hline & 70 & 39 & 18 & 22 \\
Erica arborea (\%) & 11 & 6 & 7 & 4 \\
Erica scoparia (\%) & 0 & 18 & 36 & 46 \\
Cistus monspeliensis (\%) & 1 & 9 & 14 & 8 \\
Cistus salviaefolius (\%) & 11 & 4 & 0 & 0 \\
Phillyrea angustifolia (\%) & 7 & 17 & 18 & 19 \\
Pistacia lentiscus (\%) & 0 & 7 & 7 & 2 \\
Lavandula stoechas (\%) & & 396 & 1610 & 3025 \\
Volume total (m3/ha) & 2327 & & & \\
\hline
\end{tabular}

$1986: 3$ ans après le débroussaillement d'ouverture; $1987: 1$ an après le premier débroussaillement d'entretien. 
seuil se situe aux alentours de $2000 \mathrm{~m}^{3 /}$ ha de volume d'encombrement (Étienne et al, 1989). Il est alors intéressant de calculer, à partir des courbes de régression, le temps mis par chacun des faciès pour atteindre ce seuil. Le développement de l'ériçaie et des maquis à cytise ou à calycotome exige d'intervenir tous les 2 ans et demi à 3 ans. Ensuite, la périodicité des débroussaillements s'échelonne graduellement entre 3 et 4 ans pour les faciès à myrte et la cistaie non améliorée. Enfin, le port prostré de la callune soumise à des coupes fréquentes conduit à un délai de retour se situant aux alentours de 5 ans.

Nous avons volontairement laissé de côté le cas des cistaies sursemées et fertilisées, car elles constituent une situation encore peu commune sur les réseaux de pare-feu. Toutefois, si la participation de troupeaux à l'entretien des zones débroussaillées se développe à l'avenir (INRACERPAM, 1990), on peut avancer que la mise en place d'un pâturage associé à des améliorations pastorales permettra de réduire notablement la fréquence des interventions. Ainsi, les cistaies dépassent le seuil de $2000 \mathrm{~m}^{3}$ dès la troisième année, alors qu'elles ne l'atteignent qu'au bout de 6 ans lorsqu'elles sont sursemées.

\section{Accroissement en volume des espèces}

Des modèles de croissance sous la forme de régression puissance (tableau $V$ ) ont été calculés pour toutes les espèces, en regroupant les faciès où chacune est présente en quantité suffisante (au moins $200 \mathrm{~m}^{3} / \mathrm{ha}$ ).

Les espèces repoussant le moins vite sont le lentisque, la bruyère à balais, le ciste à feuilles de sauge et le ciste cotonneux, avec des exposants inférieurs à 1,6. Viennent ensuite le myrte, la bruyère arborescente, le ciste de Montpellier et la callune, dont les exposants se situent entre 1,7 et 1,8 . Le cytise et le calycotome se détachent nettement du groupe, avec un exposant avoisinant 2.

Les modèles par espèces expliquent les dynamiques observées sur les taciès, sauf dans le cas des bruyères. Pour celles-ci,

Tableau V. Caractéristiques des régressions de croissance en volume pour chaque espèce.

\begin{tabular}{|c|c|c|c|}
\hline Espèce & $Y=f(\hat{a g} \theta)$ & $r$ & $n$ \\
\hline Pistacia lentiscus * & $Y=1,08 \times 1,26$ & $0,93^{a}$ & 20 \\
\hline Erica scoparia * & $Y=0,89 \times 1,34$ & $0,99 a$ & 14 \\
\hline Cistus albidus * & $Y=0,33 X^{1,44}$ & 0,78 a & 18 \\
\hline Cistus salviaefolius * & $Y=0,44 X^{1,52}$ & $0,83^{a}$ & 24 \\
\hline Myrtus communis * & $Y=1,24 X^{1,66}$ & 0,99 a & 10 \\
\hline Erica arborea & $Y=1,08 \times^{1,74}$ & 0,99 a & 8 \\
\hline Cistus monspeliensis * & $Y=0,87 \times 1,79$ & 0,97 a & 10 \\
\hline Calluna vulgaris & $Y=1,36 X^{1,80}$ & $0,98^{a}$ & 7 \\
\hline Cytisus trifiorus & $Y=1,84 X^{1,92}$ & 0,96 a & 12 \\
\hline Calycotome spinosa & $Y=1,43 \times 2,07$ & 0,99 a & 6 \\
\hline
\end{tabular}

$\mathrm{n}$ : nombre de points; $Y$ : phytovolume (m3/ha); $X$ : âge (mois); " : sur des faciès améliorés. Significatif au seuil de a: $P<0.001$. 
les courbes de régression sont fortement amorties par les données issues d'individus provenant d'autres faciès que l'ériçaie et ayant donc subi un nombre important de coupes (5 à 7 contre seulement 2 ).

\section{Vitesse de croissance}

Les différentes familles de courbe observées sur le développement en volume ou sur l'accumulation de phytomasse démontrent des stratégies de croissance contrastées, aussi bien au niveau des espèces que des faciès. Si l'on ramène les phytomasses produites ou les volumes encombrés à une unité de temps donnée, on obtient des vitesses de croissance qui quantifient bien la rapidité de colonisation de l'espace.

\section{Vitesse d'accumulation de phytomasse}

La figure 1 montre comment la croissance en poids des différents faciès mesurés s'effectue selon des rythmes particuliers. Certains faciès s'installent lentement puis

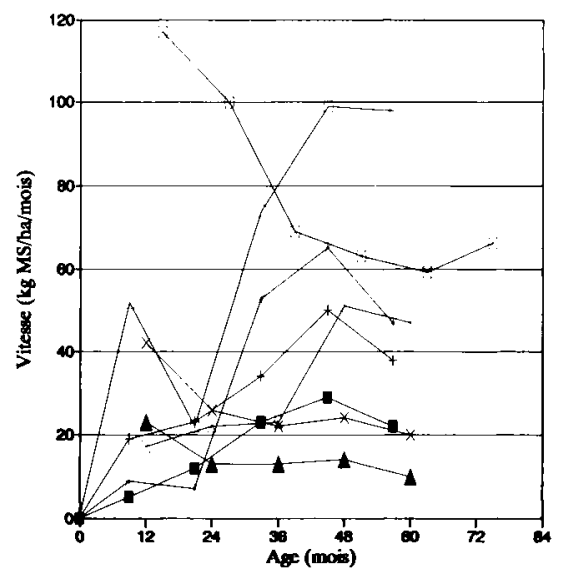

Fig 1. Vitesse de croissance en poids de 8 faciès arbustifs dans les forêts de l'Esterel. - ciste clair;- + ciste dense; * lavande ciste; $\boxminus$ myrte filaire; $*$ cytise; - - cytise fougère; $\varangle$ calycotome; $-\otimes-$ callune accélèrent leur accumulation de phytomasse entre 2 et 4 ans (cistaie, myrtaie, maquis à calycotome). D'autres au contraire démarrent très vite la première année puis diminuent leur vitesse de production progressivement (landes à callune, peuplements de cytise). II est intéressant de remarquer que différentes stations d'un même faciès suivent des courbes tout à fait similaires, avec un écart presque constant lié aux différences initiales de densité ou de recouvrement entre les stations (fig 2). Il est même possible après application d'un facteur correcteur lié à la densité initiale du peuplement, de modéliser la vitesse d'accroissement d'un faciès donné. Par exemple, pour les cistaies ou les peuplements à cytise, on obtient :

$$
V_{p}=0,46 a^{1,05}+c \quad(r=0,95)
$$

avec $c=15$ pour la cistaie dense et $c=0$ pour la cistaie claire

$$
V_{p}=95,15 a^{-0,54}+c(r=-0,90)
$$

avec $c=10$ pour le cytise dense et $c=0$ pour le cytise clair

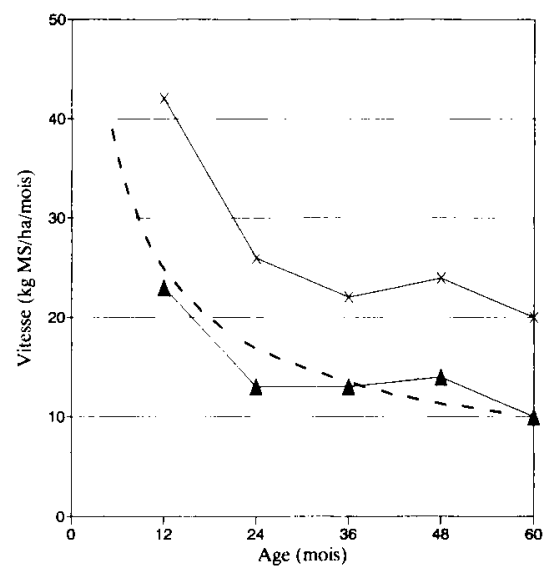

Fig 2. Modèle de croissance en poids des faciès à Cytisus triflorus; $*$ cytise; $\rightarrow$ cytise fougère. 
où $V_{p}$ est la vitesse de croissance en phytomasse exprimée en $\mathrm{kg}(\mathrm{MS}) \cdot \mathrm{ha}^{-1} \cdot \mathrm{mois}^{-1}$ et a l'âge du peuplement, en mois.

En valeur brute, les vitesses maximales varient entre 100 et $130 \mathrm{~kg}(\mathrm{MS}) \cdot \mathrm{ha}^{-1}$. mois $^{-1}$ et sont le fait des maquis à bruyère ou à myrte et des landes à callune. A l'opposé, les cistaies et les peuplements de cytise sont les plus lents à se développer, avec des vitesses inférieures à $30 \mathrm{~kg}$ (MS) $\cdot$ ha $^{-1} \cdot$ mois $^{-1}$.

Si l'on fait abstraction des à-coups mesurés pendant la phase de réinstallation du groupement végétal, les faciès se distinguent également en stabilisant leur vitesse de développement à des allures contrastées. La lande à callune se stabilise autour de 60 à $70 \mathrm{~kg}(\mathrm{MS}) \cdot \mathrm{ha}^{-1} \cdot \mathrm{mois}^{-1}$ après 40 mois, alors que les peuplements de cytise stagnent autour de $10-20 \mathrm{~kg}(\mathrm{MS}) \cdot \mathrm{ha}^{-1}$. mois $^{-1}$ dès la deuxième année (fig 1 ).

\section{Vitesse d'encombrement de l'espace}

On retrouve les mêmes allures de courbes pour les vitesses d'encombrement de l'espace. Les groupements végétaux dominés par le cytise ou la callune atteignent très rapidement des vitesses élevées (70-130 $\mathrm{m}^{3} \cdot \mathrm{ha}^{-1} \cdot \mathrm{mois}^{-1}$ ), puis se stabilisent autour de 60 pour les cytises contre 40 pour la callune. Les autres peuplements arbustifs accélèrent progressivement leur vitesse d'encombrement en passant de 10-25 $\mathrm{m}^{3} \cdot \mathrm{ha}^{-1} \cdot \mathrm{mois}^{-1}$ dans les cistaies, 30 à 60 dans les maquis à myrte et de 50 à 90 dans les peuplements de calycotome.

II est également possible de modéliser la courbe de vitesse d'encombrement d'un faciès donné. Par exemple, pour les cistaies ou les peuplements à cytise, on obtient :

$$
V_{v}=0,36 a^{0,97}+c(r=0,94)
$$

avec $c=10$ pour la cistaie dense et $c=0$ pour la cistaie claire

$$
V_{v}=320,5 a^{-0,48}+c(r=-0,81)
$$

avec $c=9$ pour le cytise dense et $c=0$ pour le cytise clair

où $V_{v}$ est la vitesse de croissance en phytovolume exprimée en $\mathrm{m}^{3} \cdot \mathrm{ha}^{-1} \cdot \mathrm{mois}^{-1}$, et a l'âge du peuplement, en mois.

On peut remarquer que ces modèles présentent un écart important avec les dérivées des équations de régression décrivant l'accroissement (tableau I). Cette différence est due d'une part, au poids de la première année de repousse dans la régression et d'autre part, à la combinaison de 2 stations dans les modèles de vitesse. Ces aspects seront analysés dans un futur article prenant en compte un grand nombre d'échantillons pour un même faciès.

\section{DISCUSSION}

Peu d’études du même genre ont été menées jusqu'à ce jour sur les peuplements arbustifs méditerranéens. Seules quelques données d'accumulation de phytomasse en fonction de l'âge sont disponibles sur les garrigues du Languedoc (Trabaud, 1990), les cistaies de Corse (Étienne, 1977b; Feuillas, 1979; Ferrando et Giacometti, 1986) ou d'Andalousie (Merino et al, 1990) et sur les ériçaies de Corse (Feuillas, 1979; Ferrando et Giacometti, 1986; Mesleard, 1987). Toutefois, les résultats présentés concernent généralement des études synchroniques dans lesquelles l'effet du substrat, de la pente et de l'altitude est au moins aussi fort que celui de l'âge du peuplement. De plus, le pas de temps observé est beaucoup plus long que le nôtre. 
On peut quand même signaler que les ordre de grandeur pour les réinstallations de cistaies après incendie se situent aux alentours de 1-1,5 TMS•ha-1 $\cdot$ an $^{-1}$ sur les 15 premières années. En comparant cette donnée avec les résultats obtenus après débroussaillement, on peut avancer que la dynamique suit le même processus puisque l'on récupère 3 TMS ligneuse au bout de 2 ans sur les pare-feu simplement débroussaillés.

Par contre, il existe une abondante littérature sur les landes à callune, avec de nombreuses données sur l'accumulation de phytomasse dans le temps après feu (Chapman et al, 1975; Merino et al, 1990), fauche (Clément et Touffet, 1976) ou arrêt du pâturage (Loiseau et Merle, 1981; Doche, 1990). Les données provenant du Royaume-Uni et du Massif central fournissent des valeurs élevées de phytomasse dès les premières années, avec une phytomasse aérienne atteignant 4 à 5 tMS) $h^{-1}$ au bout de 3-4 ans (Robertson et Davies, 1965; Barclay-Estrup, 1970; Chapman et al, 1975; Doche, 1990). Toutefois, il n'est pas possible de savoir si cette productivité, nettement supérieure à celle observée dans notre région, est liée à une meilleure adaptation écologique de la callune aux milieux non méditerranéens, ou bien à une différence de couvert arbustif entre les peuplements. Cependant, si l'on fait l'hypothèse que les landes à callune des régions océaniques ou tempérées frôlent les $100 \%$ de recouvrement, on peut constater que les productions annuelles enregistrées sont très proches des phytomasses corrigées obtenues dans l'Esterel.

De toute façon, nous avons établi un premier référenciel permettant de mieux comprendre la repousse de quelques peuplements de petits ligneux au cours de leur première phase de croissance. Les modèles proposés fournissent un premier outil d'aide à la décision dans la programmation de travaux de débroussaillement en milieu forestier. II faudra les affiner en essayant de trouver des modèles non linéaires expliquant mieux les phases successives d'accroissement, et en développant un modèle global par type de peuplement, en $y$ intégrant une fonction continue du recouvrement de la strate arbustive. II reste également à élargir la gamme des formations végétales, en essayant en particulier de développer le même type de recherche sur les formations arbustives poussant sur sol calcaire ou marneux.

\section{REFERENCES}

Barclay-Estrup P (1970) The description and interpretation of cyclical processes in a heath community. II Changes in biomass and shoot production during the Calluna cycle. $J$ Ecol 58, 243-249

Baudin F (1985) Phytovolumes, phytomasses et stratégies d'occupation spatiale de six espèces arbustives sur des pare-feu de l'Esterel. DEA écologie, Marseille, $32 \mathrm{p}$

Chapman S, Hibble J, Rafarel C (1975) Net aerial production by Calluna vulgaris on lowland heath in Britain. $J E c o l 63$ (1), 233-252

Clément $B$, Touffet $J$ (1976) Biomasse végétale aérienne et productivité des landes des monts d'Arrée (Bretagne, France). Oecol Plant 11 (4), 345-360

Combier N (1990) Consommation d'espèces arbustives méditerranéennes par un troupeau ovin sur pare-feu. Mémoire ENITA, ClermontFerrand, $42 \mathrm{p}$

Doche B (1990) Les successions articulées autour des landes à Calluna vulgaris dans le Massif central français. Comparaisons avec quelques autres systèmes à Ericacées. Bull Ecol 21 (1), 43-50

Étienne M (1977a) Un essai d'amélioration des ressources pastorales de la végétation en Corse. Fourrages 71, 83-92

Étienne M (1977b) Bases écologiques pour le développement des ressources pastorales en 
Corse. Thèse écologie, USTL Montpellier, $203 p$

Étienne M, Legrand C (1991) A non destructive method for evaluating shrubland biomass and combustibility

Étienne M, Napoleone M, Jullian P, Lachaux M (1989) Élevage ovin et protection de la forêt méditerranéenne contre les incendies. Etud Rech Syst Agr Dév 15, 1-46

Ferrando J, Giacometti N (1986) Méthodes d'évaluation de la phytomasso épigée de trois espèces : Cistus monspeliensis L, Erica arborea L, Arbutus unedo L. Deux exemples d'application. Mémoire MST, Université de Corse, $38 \mathrm{p}$

Feuillas D (1979) Méthodes et techniques d'estimation de la biomasse végétale épigée des formations arbustives et leurs applications au maquis corse dans la vallée du Fango (Corse). DEA écologie végétale, université Paris-Sud, $51 \mathrm{p}$

Forgeard F (1977) Biomasse et productivité primaire de quelques landes armoricaines. Bot Rhed A15, 29-35

INRA-CERPAM (1990) Espaces forestiers, élevage et incendie. Rev For Fr $42\left(n^{\circ}\right.$ special), 156-172

Joffre R, Casanova JB (1983) Le développement des ressources fourragères des parcours en Corse de l'intérieur. Fourrages 93, 51-84

Johnson A, Abrahamson W, McCrea K (1986) Comparison of biomass recovery after fire of a seeder (Ceratiola ericoidea) and a sprouter (Quercus inopina) species from South-central Florida. Am Midl Nat 116 (2), 423-428

Keely J, Zedler P (1979) Reproduction of chaparral shrubs after fire: a comparison of sprouting and seeding strategies. Am Midl Nat 99 (1), 142-161

Loiseau P, Merle G (1981) Production et évolution des landes a callune dans la région des Dômes. Oecol Applic 2 (4), 283-298

Merino O, Martin M, Martin A, Merino J (1990) Successional and temporal changes in primary productivity in two mediterranean scrub ecosystems. Acta Oecol 11 (1), 103-112

Mesleard F (1987) Dynamique, après perturbations, de peuplements de deux éricacées en Corse. Thèse écologie, USTL Montpellier, $146 \mathrm{p}$

Robertson R, Davies G (1965) Quantities of plant nutrients in heather ecosystems. $J$ Appl Ecol 2, 211-219

Rousseau S, Loiseau P (1982) Structure et cycle de développement des peuplements à Cytisus scoparius dans la chaîne des Puys. Oecol App/ 3 (2), 155-168

Trabaud $L$ (1990) Feu et potentialités pastorales en garrigue de chêne kermès. Fourrages 122, 175-187

Vinck N (1987) Effet du recépage et du pâturage sur les peuplements arbustifs. Mémoire ing agronome, UL Bruxelles, $204 p$ 